\title{
SUBJECTIVE MEASURES OF WELL-BEING FOR DEVELOPING COUNTRIES
}

\author{
Laura Camfield \\ ESRC Research Group on Well-being in Developing Countries, \\ University of Bath, Great Britain
}

\begin{abstract}
The paper explores the conceptual and methodological issues entailed in using subjective measures of well-being in developing countries. In the first part I define, situate and contrast subjective quality of life (QoL), subjective well-being (SWB), and well-being. I also look at the conceptual and methodological shortcomings of subjective measures of well-being and suggest ways of overcoming these by combining different approaches. I then explore how an expanded concept of subjective quality of life fits into the theoretical framework of the UK-based Well-being in Developing Countries study (or WeD), specifically how it plans to produce a new, "development-related" profile of quality of life, drawing on the methodology of the WHOQOL group $(1995 ; 1998)$.
\end{abstract}

\section{INTRODUCTION}

This paper addresses the conceptual and methodological issues entailed in using subjective measures of well-being, especially outside the Euro-American context in which they were developed. Initially I look at definitions and relationships between key terms such as subjective quality of life (QoL), subjective well-being (SWB), and well-being. I then examine the conceptual and methodological shortcomings of subjective measures of well-being and suggest ways of overcoming these by combining different approaches to investigating and measuring quality of life. The final part of the paper explores how an expanded concept of subjective quality of life fits into the theoretical framework of the UK-based Well-being in Developing Countries study (or WeD) ${ }^{1}$. I describe how our research will "draw insights from the psychological literature on subjective well-being and quality of life" and "explore how these approaches can add to our understanding of development processes" [WeD Research statement 2003]. I also explain how the quality of life research will "seek to reflect local understandings in constructing a new, "development-related" profile of quality of life" [2003], provisionally called the "DevQoL", using a simplified version of the definition of quality of $\operatorname{life}^{2}$ and "spoke-wheel" methodology developed by the Quality of life group of the World Health Organization (WHOQOL 1995; 1998).

1

Wolfgang Glatzer, Susanne von Below, Matthias Stoffregen (eds.), Challenges for the Quality of Life in Contemporary Societies, \#\#\# \#\#\#.

(C) 2004 Kluwer Academic Publishers. Printed in the Netherlands. 


\section{SUBJECTIVE QUALITY OF LIFE}

I prefix quality of life with "subjective" to distinguish my understanding of it as a subjectively valued experiential state ("to have good quality of life") from its more common use as an objective composite indicator. Like health (another notoriously difficult concept to define), quality of life can be simultaneously an evaluation, a state, a goal, and an indicator, depending on context. Measuring subjective quality of life should not only involve "self-report" by the subject in response to direct questions about their quality of life, but should also enable them to indicate the importance to them of each aspect of life or more radically, specify the aspects that they consider most influential on their quality of life.

\section{SUBJECTIVE QUALITY OF LIFE IN HEALTHCARE}

Although subjective quality of life measures originated in social indicators research in the mid 1970s (e.g. Andrews and Withey's General Well-being Scale 1976), they have been used most extensively within healthcare. Quality of life measures have grown exponentially over the past decade, funded by national health services, pharmaceuticals and the US health insurance industry. Medline, the main index for medical papers, first used the phrase "quality of life" as a heading in 1975. Since then tens of thousands of papers have been published, nearly 18,000 between 2000 and 2003 alone, and there have been a proliferation of study groups, conferences and special journal issues. This remarkable growth can be linked to increasing cost consciousness in medicine, risk management by health care providers, and the need for more sensitive measures to compare treatments for chronic illness. The same pressures operate on development practitioners and their funders who need to focus their activities on demonstrably effective interventions, which have minimal negative impact on their host communities. While donors are not quite at the stage of using "Happiness-adjusted Life Years" to decide which projects to fund (Veenhoven, $2003)^{3}$, simple tools for assessment and evaluation are needed to supplement conventional economic indicators.

There are three types of subjective quality of life measure used in healthcare:

generic (or universal), which can be used in sick and "healthy" populations, cover all conditions, and are brief and easier to translate (for example, the MOS Short form 36-item health survey or SF-36 [Ware, 1994]);

disease-specific (or local, referring to the concerns of a specific "imagined community"), which are generated from interviews with clinicians and people with particular conditions and are only appropriate to that condition; and

individualized, where individual respondents specify the areas of life that are important to them and evaluate their performance in those domains (for example, the Patient Generated Index or PGI [Ruta et al., 1994]). ${ }^{4}$

Although disease-specific measures are obviously relevant, generic measures are widely used as they express outcomes in a standard numerical format. This makes it possible to compare the quality of life of people with different conditions and, by combining the scores with clinical information and population preferences, to estimate the impact of different conditions and make ostensibly participatory decisions about funding priorities using metrics like the quality-adjusted life year. Individual- 
ized measures are rarely used in either healthcare or health services research, however, they offer an interesting model for our research as they are designed both to "elicit the value system of individual respondents and to quantify quality of life using this elicited system" (Browne et al., 1997, p. 742. quoted in Kavita, et al., 2003). They also enable us to investigate whether subjective quality of life is simply "the extent to which our hopes and ambitions are matched by experience" (Calnan, 1984).

\section{MEASURING SUBJECTIVE QUALITY OF LIFE: METHODOLOGICAL CHALLENGES}

Researchers can use quality of life very loosely: One popular edited collection entitled Quality of Life after Open-heart Surgery focused on common medical outcomes like clinical measures and survival times. Descriptions of the measures like quality of life, health status, or well-being are used interchangeably, even though they have different histories and problems. For example, "heath-related quality of life" which is the term currently in vogue in healthcare implies that quality of life can be separated into health and non-health related components. ${ }^{5}$

Although quality of life is defined as a holistic concept, namely what a person feels about their life when they evaluate it as a whole, this is not necessarily what quality of life measures assess as they are biased towards "physical function" (e.g. Leplege \& Hunt, 1997), despite the fact that population and patient surveys suggest it is not the most important determinant of quality of life (e.g. McDowell \& Newell, 1987; Bowling, 1995). Valued aspects of life like feeling a sense of identity or belonging have been excluded for the pragmatic reason that the majority would not respond to medical interventions. ${ }^{6}$ In the case of the WHOQOL, this would have prevented it from being used in international clinical trials, which was an important objective for the most powerful of its user groups. Another reason for exclusion is the tendency for measures to replicate themselves: Many "new" items come from reviews of existing measures which new measures are then validated against ${ }^{7}$ (c.f. Hacking, 1995, on the tautologousness of measures of multiple personality disorder). Measures therefore need to be different enough to be perceived as new, but not so different that other measures cannot be used to "validate" them or contextualize their results.

Even studies undertaken from the individualistic perspective of biomedicine suggest the main determinants of quality of life are social and environmental. This is obscured, however, when quality of life is located solely in the "natural" body of the individual. A regression analysis of mean happiness scores from eleven quality of life surveys carried out by Michalos (2001) found that satisfaction with health was never the strongest predictor of happiness and in five studies failed to qualify for the final regression due to lack of statistical significance. Although self-reported health (measured with the SF-36) accounted for $4 \%$ of explicable variance in happiness scores, this was entirely due to the scores on the mental health domain. ${ }^{8}$

We might also ask how the individualistic focus of the majority of quality of life measures affects their comprehensibility in societies that operate with different models of the person, for example, what is characterized as internal or external to a 
person. On a purely practical level, many of the normative reference points for functional scales may be irrelevant; for example, the majority of the functional items in French blindness scales (e.g., "Can you drive a car?", "Can you see street lamps?”) needed to be reworked ("Do you know which way is east?" -necessary to establish orientation for prayer, "Do you fall into holes?"), so they could be used in francophone Mali (Leplege et al., 1999). Problems of translation go beyond vocabulary to the tacit models underlying the measures, which exemplify the operation of Lukes's second and third levels of power (1986). The models not only set the agenda but shape the ways in which the issues can be thought about in the first place; for example, the Cartesian dualism of separate physical and psychological domains and the way the social domain, if it has been included, is represented as external to the person. The veteran quality of life researcher Hunt observes ironically that "the ethnocentricity of assuming that a measure developed in, say, the USA, or England, will be applicable (after adaptation) in pretty much any country or language in the world (...) is highlighted if one imagines the chances of a health questionnaire developed in Bali, Nigeria, or Hong Kong, being deemed suitable for use in Newcastle, Newark, or Nice" (1999). Bearing in mind the way these methodological issues multiply when quality of life measures are used cross-culturally, we might ask what is driving the international expansion of quality of life measurement?

\section{MEASURING SUBJECTIVE QUALITY OF LIFE CROSS-CULTURALLY: THE WHOQOL MEASURE}

Internationally, the World Health Organisation or WHO has been one of the keenest advocates of quality of life, which is consonant with its definition of health as "a state of complete physical, mental and social well-being, and not merely the absence of disease or infirmity (...) whose realization requires the action of many other social and economic sectors". In 1991 the WHO formed the WHOQOL group to develop a measure that would assess quality of life, rather than merely the impact of disease and impairment, perceived health, or functional status, as existing "quality of life" measures did. This measure would combat "the increasingly mechanistic model of medicine" and use quality of life assessments to introduce a "humanistic element into healthcare", supporting the WHO's "continued promotion of an holistic approach to health and healthcare".

The WHOQOL's "spoke-wheel" development process, where a common and consensually derived methodology enabled it to be developed simultaneously in 15 countries, is now recognized as the gold standard for international projects (WHOQuality of Life, 1998). However, it is a resource-intensive process that requires access to translation and transcription facilities, which may not be available in most developing countries. ${ }^{9}$ The original WHOQOL process was also not fully participatory as the six domains of quality of life were defined in advance by a small group of "experts", setting the agenda for the population focus groups who generated the items. Although there was some iteration between the advisory group and the focus groups, the structure of the instrument did not change substantially (a domain with a single facet was added on spirituality, religion and personal beliefs) and continued to reflect similar measures in the field. We plan to approach the topic as openly as 
possible with a diverse sample of respondents from the rural and urban sites where we are working. The researchers will use a range of qualitative methods, including semi-structured interviews with individuals and groups and participatory techniques, and will already have spent some time in the site building relationships with local people. The findings of the exploratory fieldwork will be combined with data from the country workshops on quality of life ${ }^{10}$ to create a "quality of life framework", which will be discussed with "focus-groups" of local people and development practitioners. Although focus group research has been criticized for lack of representativeness and power imbalances, these can be surmounted by choosing an appropriate activity (e.g. exploring areas of consensus rather than difference), sampling carefully, and using skilled and reflexive facilitators. Historically the qualitative contribution to measure development has been neglected, possibly due to researchers' discomfort with the less scientific aspects of the process. We plan to use the expertise of the WeD team in qualitative methods (and their field experience) to ground the DevQoL, enabling it to provide a more nuanced analysis of people's lives.

We will get people to prioritorize aspects of quality of life using either the methodology of the WHOQOL-100 or the Person Generated Index ${ }^{11}$, depending on which works best in the field. This will enable us to measure what effect a gap between people's aspirations and achievements has on their evaluations of quality of life, a theme already identified as important by researchers like Michalos (1985), Calnan (1984) and Skevington et al. (Skevington \& O'Connell, 2003).

Despite the apparent comprehensiveness of the 24 WHOQOL facets, which range from self-esteem to transport, the WHOQOL-100 presents a fragmented and disembodied view of life. It clearly needs strengthening in areas like economic, political and financial relationships, security, and social inclusion; omissions confirmed by our theoretical frameworks and empirical data from the quality of life workshops. Working from the WHOQOL model in the way we have described will hopefully enable the DevQoL to fulfill the promise of the WHOQOL group's original definition by setting individual perceptions of quality of life in their cultural and social context.

The observations we have made about the WHOQOL apply with greater force to other quality of life measures in its field. But is it surprising that measures designed by western psychologists for use by doctors and economists should be individualistic, non-political, and curiously disembodied? Having discussed the slightly different use of quality of life measurement within healthcare, in the next part of the paper, I will define SWB and Well-being, examine conventional indicators of life satisfaction and happiness, and draw some methodological conclusions.

\section{SUBJECTIVE WELL-BEING (SWB)}

SWB is usually defined as a subjective measurement that combines the presence of positive emotions and absence of negative emotions with overall satisfaction with life (Diener, 1984). As with quality of life, however, measures of SWB can present a different picture. For example, Cummins includes subjective and objective measures of material wellbeing, health, productivity, intimacy, safety, place in community, and emotional well-being (Cummins, 1997), which may be why it has been de- 
scribed as a measure of "need satisfaction" rather than SWB. ${ }^{12}$ Ryff's six factor measure of SWB is similarly expansive, though possibly more culturally specific ${ }^{13}$, comprising self-acceptance, personal growth, purpose in life, positive relations with others, autonomy, happiness and environmental mastery (Ryff, 1995).

SWB is most commonly measured using self-report questions about happiness or life satisfaction, the results of which have become increasingly important to the social indicators movement, "economists of happiness" like Clarke and Oswald, and policy makers (e.g. the recent Cabinet Office report on the implication of "life satisfaction" for UK government policy, Strategy Unit, 2002). In the next part of the paper I explore some of the problems with standard measures of happiness and life satisfaction and link my critique back to the WHOQOL through its characterization as a measure of life satisfaction rather than quality of life.

\section{HAPPINESS AND LIFE SATISFACTION}

Standard questions about happiness and life satisfaction ${ }^{14}$ are a routine part of data sets like the World Values Survey (http://wvs.isr.umich.edu/), the Eurobarometer (http://europa.eu.int/comm/public_opinion/), and the South African General Household survey (http://www.statssa.gov.za/) and have been collated for comparative purposes in the World Database of Happiness (http://www.eur.nl/fsw/research/ happiness/). There are, however, some problems with using this data. Firstly, the phrasing of the questions and the response scales used were not uniform across countries and time periods, which may have affected responses. Secondly, "global" questions on subjective well-being (e.g. "Taking everything as a whole, how is your quality of life?") are more prone to cognitive or mood biases than domain specific ones (Schwartz \& Strack, 1999) and may also be very difficult to answer sensibly! We hope to avoid this problem by making the questions sufficiently specific to ensure that they are comprehensible, meaningful and can be answered accurately. It is more useful to know that people are unhappy with their working conditions, or that they were happier before their employer used a loan from a micro-credit scheme to install unsafe machinery, than it is to know their level of satisfaction overall, whether this is measured on a 5,11 or 100 point scale!

Although positive and negative feelings represent only two of the 24 domains of the WHOQOL, half the items are phrased in terms of satisfaction (for example, "how satisfied are you with your sleep?"), possibly due to the fact that the English language offers a limited number of ways to ask people to evaluate aspects of their life. This prompted the quality of life researchers Williams (2000) and Hagerty (Hagerty et al., 2001) to controversially suggest it be compared with measures of life satisfaction rather than quality of life. This is not necessarily a compliment. Although life satisfaction has been widely used as a more "cognitive" (and thus scientific) measure of SWB than happiness ${ }^{15}$, the concept has its own problems: The coordinator of a project investigating well-being in the UK stopped asking people how satisfied they were because the word sounded "dead" and provoked responses like "well, I mustn't grumble..." (Nick Marks, New Economics Foundation, personal communication). Similarly, initial research by the WeD teams in Thailand and Peru questioned the meaningfulness of the concept when it related to circumstances 
that people could not control or change, as their only option was satisfaction (personal communication). Life satisfaction becomes redundant in situations where people have little control or choice in the areas that matter to them and unhelpfully evokes Western discourses of voluntarism and consumer power.

While the word satisfaction is described as universally meaningful, this does not mean it has the same meaning universally: Killian's review of "patient satisfaction" studies in the UK described how being satisfied with a treatment can encompass feelings of resignation and helplessness and a belief that the treatment is useless (Kilian et al., 1999). Recent psychological research (Schimmack et al., 2002) also suggests a personality "trait-level" propensity to be satisfied with life is a more important determinant of life satisfaction scores than objective life circumstances, which may reduce its utility as a social indicator. This is an interesting addition to the studies of identical and non-identical twins carried out by Hamer (1996) and Lykken and Tellegren (1996) which measured the happiness of twins raised apart and together and concluded that up to $80 \%$ of the stable differences in life satisfaction were heritable. Less than $3 \%$ of variance was explained by socio-economic status, educational attainment, family income, marital status and religious commitment.

Although being happy and/or satisfied with life are intrinsically valuable states, and ones that have been neglected in development (Clark, 2000), we cannot presume that they have the same meaning or priority in every country or situation. Consequently, a measure is needed that assesses the presence of both locally valued experiential states and the fulfillment of more prosaic needs for material, relational, and cultural resources. This state of optimum experiential and need satisfaction we have provisionally called "development-related quality of life", drawing on the WeD group's stipulative definition of "development" as interventions aiming to produce a state where the needs that people value are satisfied. The prefix "developmentrelated" functions in the same way as "health-related" quality of life to focus our attention on the aspects of quality of life that will be affected by development projects, recognizing that their effects may be multi-dimensional and unintended. Perhaps an exploration of the comparatively uncontested concept of well-being can help us move towards a better understanding of how development-related quality of life should be defined and measured?

\section{WELL-BEING}

The "constituent elements" of well-being or a good form of human life have been understood respectively as satisfaction of desires or preferences, prudential values (arguably the human desire account taken to its logical limit), positive freedoms, and human capabilities or needs. All these accounts present certain problems, for example, desires may be other-directed, ill informed, adaptive, or not reflect the person's values. While certain shared values or concepts of quality of life are necessary for mutual intelligibility, they do not comprise a comprehensive vision of good quality of life. Nor can their priority be assumed in any situation; I am reminded of an Angolan child refugee who when asked how it felt to be chased from her home by a murderous gang - yes, journalists do ask these sort of things - said that the worst 
part was losing her one good set of clothes because she now could not appear in public without shame. This kind of understanding (what Saltmarshe calls "seen from the corner of the eye", 2003) is highly valued by qualitative researchers. The need to avoid foreclosing on it inspired Clark's open-ended questionnaire for exploring poor South Africans' conceptions of a good life $(2000)^{16}$, which may be included within the WeD project. Although the lists it produced closely resemble lists of basic needs or core values, the process also produced some surprises. For example, the priority given to Coca-Cola ${ }^{\mathrm{TM}}$, offered a greater understanding of the rationality underlying people's choices through an exploration of the many different functionings this product could support. ${ }^{17}$

While Subjective Quality of life and SWB are often treated as interchangeable, I argue that Subjective Quality of life actually has more in common with well-being. Both concepts are multi-dimensional, context specific, and incorporate things people have reason to value, as well as their wants and needs. The similarity is apparent when we compare Clark's definition of well-being as "the constituent elements of a good form of human life - whatever that may be" (2000) with the WHOQOL group's definition of quality of life as "an individual's perceptions of their position in life in the context of the culture and value systems in which they live and in relation to their goals, expectations, standards and concerns" (1995). They differ only in two respects: firstly, Clark's method for measuring well-being reflects his foundational definition in a way that the WHOQOL-100 does not. For example, unlike individualized measures of quality of life like the Schedule for the Evaluation of Individual Quality of Life (SeiQoL) (O'Boyle et al., 1992) or Patient Generated Index (PGI) (Ruta et al., 1994) the WHOQOL -100 does not allow people to specify their concerns or goals although they can indicate priority among the choices offered to them.

The reasons for this are pragmatic - setting parameters for people's responses ensures the process is quick, comparable, easy to analyze, and more "scientific", especially if you believe people cannot reliably articulate what is important to them. Secondly, Clark's definition does not explicitly acknowledge the cultural and structural constraints on people's conception of the good life, or the potential tension between the individual and the collective if a good life for one individual involves exploiting or abusing another. The additional elements of the WHOQOL definition, for example, the role of social norms in shaping people's expectations, aims, perceptions of success and failure, and overall evaluations of quality of life, may be best explored qualitatively. ${ }^{18}$

\section{COMBINING APPROACHES TO MEASURING SWB}

I suspect that the elision of subjective Quality of life and SWB has occurred for two reasons; firstly, similarities in their language of measurement, which I describe below. Secondly, both are perceived as the domain of psychologists, which may account for their individual and ethnocentric focus. Current debates over measurement have obscured the theoretical distinctions between Quality of life and SWB, due to what Veenhoven describes as their "domination by psychometricians, who focus [...] on factor loadings, reliability issues and inter-test correlations" at the expense 
of a "clear answer to the question of what these measures actually measure" (Veenhoven, 2000, pp. 19-20).

Some theorists from quality of life and SWB backgrounds have also attempted to bring the two concepts into a fruitful relationship. For example, Kahneman, Diener and Schwarz in their preface to "Hedonic Psychology" (1999). Kahneman et al. suggest that analyses of quality of life should include cultural and social context, individual values, capabilities and objective circumstances, and SWB. They claim that "any evaluation of quality of life is embedded in the cultural and social context of both the subject and the evaluator [...and] cannot be reduced to the balance of pleasure and pain, or to assessments of subjective life-satisfaction" (ibid., p. X). However, they place experiences of pleasure and SWB "at the centre of the story" (undoubtedly influenced by their disciplinary and cultural backgrounds), which may not be true of less hedonistic societies.

Veenhoven also observed little consensus over the meaning of quality of life and well-being (2000). Meanings are often confined to their own discursive communities, there is marked divergence between scientific definitions and common usage, and the inclusiveness of the terms makes them difficult to operationalize. He attempts to overcome this by restating the fundamental questions of "what is quality?" and "whose life quality are we talking about?" He divides quality of life into opportunities and outcomes and external and internal, for example, environmental "livability" and personal "life-ability" or capabilities. Veenhoven also distinguishes between the objective utility and subjective appreciation of life, basically its social meaning or significance versus the degree of felt satisfaction or happiness. He admits that the former is more difficult to measure quantitatively than the latter, especially as in the short-term individual happiness is a very poor indicator of social utility!

\section{USING MEASURES OF SUBJECTIVE WELL-BEING IN THE WED FIELD SITES}

In the remainder of the paper I argue that, especially for work in developing country contexts, we need to develop a measure that combines the most useful concepts and methods from Quality of life, SWB and well-being literatures. This would be based on a more expansive and actor-oriented conception of Quality of life and supplemented by extensive, in-depth qualitative work. This is essential not only so that the measure will be meaningful to its respondents but also so the data will be meaningful to the analysts. For example, curves plotted with WHOQOL data from different countries tend to show that responses to the items are concentrated around points 3 and 4 of the 5 point response scale, "neither satisfied nor dissatisfied" and "satisfied" (e.g. WHOQOL Group, 1998). While this could mean that the WHOQOL represents dimensions that are common to all humans, another interpretation has been offered by Bourdieu in his critique of methods of surveying opinion (1989). He suggests this represents nothing more than the human tendency to circle the middle number in a questionnaire, particularly when you are uncertain what is being asked or for what purpose the data will be used. This finding could also support the characterization of the WHOQOL as a life satisfaction measure since life satisfaction 
measures have a similar response pattern of means of $75+2.5$ in western nations and $70+5$ in non-western nations (Cummins, 2002). This may be due to the Positive Cognitive Biases described in Cummins and Nistico's homeostatic theory of SWB (2000), or less charitably, because they share the methodological shortcomings identified by Bourdieu.

In the case of the WHOQOL, this may be exacerbated by the abstract and general nature of the questions, for example, "how available to you is the information that you need in everyday life?" which were designed so they would be equally meaningful (or less) across all countries. When the questionnaire is interviewadministered to respondents who cannot read or write (the majority of participants in our study) it puts the researcher in an unenviable position since the protocol forbids them from answering the obvious question "information for what?"

The question of how much support to give to respondents is an important one on the one hand we do not want to inadvertently bias the results of the measure (producing results that would not be acceptable to our psychological peers), but on the other the interview-administration needs to be a relaxed and almost conversational interaction where respondents feel able to ask the interviewer for clarification or decide on a response category in negotiation with other household members. Sadly, the realities of field research bears little resemblance to the quasiexperimental conditions demanded by protocols for instrument administration (for example, most measures contain a check box to indicate if they have been filled in unassisted; if this has not been checked, the data is usually excluded).

This is particularly problematic for the WHOQOL's social domain as while qualitative research (e.g. Resource Profiles research in South Asia, Thailand, and Eastern Europe, cf.. McGregor, 2000) suggests it is one of the most important aspects of people's lives, the domain is psychometrically weak (O'Carroll et al., 2000; Hagerty et al., 2001). The reason for this is that it only has three facets, one of which, sexual activity, is prone to missing data even when the person is responding in the privacy of their own bedroom, rather than to an interviewer of a different gender or generation, in front of their entire household. The example highlights the fallacy of assuming that responses to the measure will be anonymous and unmediated, as would be expected in a similar study in Britain. ${ }^{19}$

Length is a more obvious problem when measures are interview administered and researchers coming from health-related quality of life can tend to over estimate the enthusiasm of respondents (I recall one study that sent pancreatic transplant recipients eleven different measures, Milde et al., 1992). People with limiting conditions are an unusually compliant and conscientious group (survey response rates average $85 \%$ ) and may have plenty of time to fill. They may also have grown up in a culture where surveys are a common form of democratic participation and selfexploration; I doubt many participants in our study will thank us for the opportunity to respond to a very personal questionnaire because they "learnt so much about themselves"! ${ }^{20}$ There is an obvious tension within WeD between creating a measure that will be more inclusive and locally relevant than anything that has gone before but will not take hours to administer. It also needs to be scientific (i.e. valid and replicable) but able to be administered in a relaxed and conversational way. In short, it needs to be an "appropriate technology". I do not believe these aims are irrecon- 
cilable, but it is important to be aware of the issues that have impeded similar projects in the past.

\section{WED'S MIXED-METHOD APPROACH TO EXPLORING SUBJECTIVE WELL- BEING}

Having explored some of the problems with the existing forms of quality of life measurement, the question still remains as to how the WeD project will investigate subjective quality of life. In order to capture the different dimensions of quality of life explored in this paper, we will be triangulating our data using a mixed methodology, or, in lay terms, covering our bets. We plan to combine detailed qualitative research with the development of a quality of life measure that is recognized as sound in quality of life circles, works well with rural respondents in developing countries, is acceptable to local development practitioners, and does not contradict our commitment to bringing together "local" and "universal" perspectives on quality of life and well-being. This commitment is theoretically as well as methodologically significant since one of the roles of the quality of life input is to facilitate communication between the universal, represented by Doyal and Gough's Theory of Human Needs (1991), and the local, represented by the Resource Profiles Approach (McGregor, 2000). The Theory of Human Needs develops a cross-cultural concept of basic needs as universal prerequisites that enable sustained participation in a chosen form of life. Its original dimensions of participation, physical health and autonomy may be extended to include psychological well-being and competences, and affiliation and belongingness (Gough, personal communication). The Resource Profiles Approach works at the local level using an actor-oriented perspective. It posits that individuals, households and communities in developing countries actively "manage" a complex of resources (material, human, social, cultural, and natural) to achieve the best possible outcome. Communication between the levels is conceptually facilitated by the notion of quality of life. The quality of life research will be attempting to produce a universal measure that is grounded in local realities, and supplement it with qualitative investigations of people's understandings of good quality of life and strategies for achieving it.

Developing the measure will involve investigating the categories and components of quality of life in the WeD field sites through semi-structured 'interactions' with a diverse range of informants and validating the emergent quality of life framework with local people and other key stakeholders. This will enable us to distinguish between components of quality of life at the universal level and those of the locality and the culture-group, and explore the relationship between them. We will also assess the level at which it is possible to produce a measure of quality of life that is meaningful to respondents, analysts and development practitioners and develop methods that will best achieve this. I will not elaborate further on the proposed methodology as this information is available in papers produced by the WHOQOL group $(1995 ; 1998)$. It has been expanded to include the new technique of "cognitive debriefing", which involves extensive interviews exploring how people interpret and respond to the measure. This will ensure that in every country we are measuring not only what we should be measuring but what we think we are measuring. We will use 
two "global" questions about happiness and life satisfaction (translated into the appropriate local terms) as these will enable interesting comparisons with existing data sets and may also adapt the Satisfaction with Life (SWLS) (Diener et al., 1985) and Positive and Negative Affect (PANAS) (Watson et al., 1988) scales.

Additionally, we plan to use a modified version of the SEIQoL or the "global" version of the PGI (now called the "Person Generated Index") (Ruta, 1997), where people would be asked to nominate the areas of their life that have most influence on their quality of life (or that of their household) and indicate their relative importance. This would be done using a common participatory technique like distributing a number of coins across the areas to represent their relative influence. ${ }^{21} \mathrm{We}$ could then ask them to assess their current status in each of these areas using the same technique. The advantage of this process is that it is quick, simple, can be carried out using participatory techniques, and, compared to a 100 item questionnaire, involves minimal translation. This means it can be repeated many times during fieldwork to explore issues of seasonality. Although the data produced will have intra rather than inter-personal comparability, it will be possible to compare the extent of the gap between expectations and achievements, which we expect to correlate significantly with SWB. It also provides a good starting point for discussions about how the respondent defines quality of life and prioritorizes the different elements within it, and why their quality of life has changed over the preceding period.

Using these approaches we hope to cover all aspects of people's subjective quality of life and communicate with people who find responding to a questionnaire or heavily structured interview an alienating experience. Objective quality of life will not be neglected, of course, and we hope to have sufficient data, combined with reflexive accounts of process, to enable a fruitful exploration of any discrepancies between the two. Perhaps by refusing to prioritize objective over subjective or qualitative over quantitative we are storing up trouble for the data analysis phase, but we hope this radical openness will enable us to learn from all the approaches used and ultimately provide methodological guidance as well as comprehensive data sets to future researchers in this field.

\section{NOTES}

1 WeD is developing a framework for studying poverty, inequality and quality of life in four developing countries (Bangladesh, Thailand, Ethiopia, and Peru).

2 "An individual's perception of their position in life, in the context of the culture in which they live" (adapted from [WHOQOL Group 1993 \& 1995])

3 These combine life satisfaction scores with life expectancy and were proposed as a possible measure at the ISOQOLS conference 2003 (see also Veenhoven, 1996).

4 Individualised measures are becoming increasingly influential within medicine because they have high 'face' and 'content' validity and directly address the changes that are important to patients (see Joyce et al [1999] for a useful review). In relation to development practice they could perform an analogous role in highlighting areas of people's lives where intervention could be focused, or warning where poorly researched interventions might damage the quality of life of the people they are intended to benefit.

5 See Michalos (2001) for a critique of this concept, which he sees as both an example of biomedical expansionism (health $=$ quality of life) and confounded (if health = quality of life, then the concept of health-related quality of life is as meaningless as "quality of life-related quality of life" would be!)

6 I observed this process during the creation of the Multiple Sclerosis Impact Scale-29 item (Hobart et al., 2001) where statements that the researchers did not think related to quality of life (e.g., reactions 
to diagnosis) were excluded from the first draft of the measure. Subsequently, items related to "coping with multiple sclerosis" and the "positive impact of multiple sclerosis" were excluded as "irrelevant", although they were obviously relevant to the people who generated them.

7 Fox-Rushby attributes this to the "nature of research careers" where "any one researcher has the opportunity to influence the development of more than one instrument, either by moving between research institutions, by sitting on different steering committees, or by becoming part of the many flourishing international groups" (for example, one WHOQOL researcher has also been involved in the IHQL and EuroQOL and another in the SIP and QWB) (1995).

8 This is good news for critics like Ravallion (2000) who argue that the effects of personality and mood make subjective measures unreliable (or for global manufacturers of anti-depressants who were quick incorporate disease rankings from the Global Burden Disease study in their marketing strategy, WHO, 2000).

9 We estimated that if we followed the WHOQOL protocol exactly, each language version of the DevQoL would require 680 hours of transcription and 920 hours of translation.

10 These were conducted in all four countries during our initial visits between June and October 2003, using a protocol piloted among Country representatives at the WeD inaugural workshop in Bath (January 2003).

11 The WHOQOL asks people to rate each item using a 5 point Likert scale, for example "How important to you is your quality of life? 1: Not important [...] 5: Extremely important" and the PGI invites them to distribute points or coins across their five priority areas.

12 It also contains an element of individual weighting through importance weightings and is usually administered alongside a measure of "national well-being".

13 Christopher (1999) makes a powerful critique of the cultural specificity of the model of well-being underlying Ryff's measure.

14 For example, "All things considered, how satisfied or dissatisfied are you with your life-as-a-whole now? 1 dissatisfied... 10 satisfied" (Veenhoven, 2001, 20022; www.eur.nl/fsw/research/happiness).

15 Cf. Veenhoven's "World Database of Happiness Research", which, despite its name, uses a standard life satisfaction question.

16 Clark's approach is an interesting mix of philosophy (fully formed conceptions of the good life can be accessed through philosophical enquiry) and inverted snobbery (ordinary people are the best judge of what constitutes a good life and it does not include opera!) There has been debate within the WeD group over whether people's values can be explored through abstract questioning, or, setting this question aside, how they translate to behavior (a point also made by critics of health economic methods for generating population valuations of different health states).

17 Unfortunately, due to the limitations of survey research on a small budget, these meanings could no be fully explored in subsequent qualitative work. This lack of follow-up has also been a problem in quality of life work where bizarre or intriguing results have not been investigated or contextualized (for example, why participants from a study using the Rosser Well-being Scale described "feeling a lack of ambition" as $45 \%$ worse than "complete bowel incontinence", Rosser et al., 1992).

18 One of the WeD team members is piloting a protocol in 21 villages in Ethiopia, which explores these themes through participatory and qualitative work with groups and individuals (for example, identifying local understandings of well-being, quality of life and poverty) (Ethiopia WeD Research Programme 2003).

19 Of course, even this cannot be assumed. During my Ph.D. I interviewed six people who had responded to a pilot questionnaire measuring quality of life in Dystonia (Camfield, 2002). Their accounts illustrated the ways questionnaires can be "destabilized" by respondents not behaving in the expected manner or engaging in "anti-programs" (Latour, 1992) like using a proxy respondent, missing out questions, answering "sarcastically", or responding on the basis of age or other conditions.

20 This interaction was described to me by a researcher on a project designing a measure of the healthrelated quality of life of older adults.

21 See Bevan et al, 2003 for an account of a 'very informal pilot' of this technique, which took place in Ethiopia during our grounding and piloting phase.

\section{REFERENCES}

Andrews, F., Withey, S. (1976): Social Indicators of Well-being: American Perceptions of Quality of Life, New York: Plenum Press. 
Bevan P., K. Kebede \& A. Pankhurst A. (2003): A Report on a very Informal Pilot of the Person Generated Index $($ ) of Quality of Life in Ethiopia (unpublished).

Bowling, A. (1995): A Survey of the Public's Judgements to Inform Scales of Quality of Life Social Science and Medicine 41, 1411-17.

Calnan, K. C. (1984): Quality of Life in Cancer Patients - A Hypothesis. Journal of Medicinal Ethics 10, 124.

Camfield, L. (2002): Measuring Quality of Life in Dystonia: An Ethnography of Contested Representations, London: $\mathrm{PhD}$ thesis, University of London.

Christopher, J. C. (1999): Situating Psychological Well-being: Exploring the Cultural Roots of its Theory and Research. Journal of Counseling and Development 77, 141-52.

Clark, D. (2000): Visions of Development: A Study of Human Values, Cheltenham: Edward Elgar.

Cummins, R. A. (1997): Comprehensive Quality of Life Scale - Adult. Manual, $5^{\text {th }}$ edition, Melbourne: School of Psychology, Deakin University.

Cummins, R. A. (2002): Normative Life Satisfaction: Measurement Issues and a Homeostatic Model Social Indicators Research 64, 225-56.

Cummins, R. A \& H. Nistico (2002): Maintaining Life Satisfaction: The Role of Positive Cognitive Bias Journal of Happiness Studies 3 (1), 37-69.

Diener, E. (1984): Subjective Well-being. Psychological Bulletin 95 (3), 542-75.

Doyal, L. \& I. Gough (1991): A Theory of Human Need, London: Macmillan.

Ethiopia WeD Research Programme (2003): Well-being and Ill-being Dynamics in Ethiopia. A Study in 20 Rural Sites (unpublished).

Fox-Rushby, J. \& M. Parker (1995): Culture and the Measurement of Health-related Quality of Life, European Review of Applied Psychology 45 (4), 257-63.

Hacking, I. (1995): Rewriting the Soul. Multiple Personality and the Sciences of Memory, New York: Princeton.

Hagerty, M. R., R. A. Cummins, A. L. Ferriss, K. C. Land, A. C. Michalos, M. Peterson, A. Sharpe, J. Sirgy \& J. Vogel (2001): Quality of Life Indexes for National Policy: Review and Agenda for Research. Social Indicators Research 20 (9), 225-40.

Hamer, D. H. (1996): The Heritability of Happiness. Nature Genetics 14 (2), 125-6.

Hobart, J., D. Lamping, R. Fitzpatrick, A. Riazi \& A. Thompson (2001): The Multiple Sclerosis Impact Scale (MSIS-29): A New Patient-based Outcome Measure. Brain 124 (5), 962-73.

Hunt, S. M. (1999): The Researcher's Tale: A Story of Virtue Lost and Regained. C. R. B. Joyce, C. A O’Boyle \& H. McGee (eds.): Individual Quality of Life: Approaches to Conceptualization and Measurement, Amsterdam: Harwood.

O'Carroll, R. E., K. Smith, M. Couston, J. A. Cossar \& P. C. Hayes (2000): A Comparison of the WHOQuality of Life-100 and the WHO-Quality of Life-BREF in Detecting Change in Quality of Life Following Liver Transplantation. Quality of Life Research 9, 121-4.

Kahneman, D., E. Diener \& N. Schwarz (1999): Preface. Kahneman, D., E. Diener, \& N. Schwarz (eds.): Well-being: The Foundations of Hedonic Psychology, New York: Russell Sage Foundation.

Kind, P., R. Rosser \& A. Williams. (1982): Valuation of the Quality of Life: Some Psychometric Evidence. M. W. Jones-Lee (ed.): The Value of Life and Safety, Amsterdam: Elsevier.

Latour, B. (1992): Where are the Missing Masses? The Sociology of a Few Mundane Artifacts. W. Bjiker, \& J. Law (eds.): Shaping Technology, Building Society: Studies in Sociotechnological Change, Cambridge, Mass.: MIT Press.

Leplege, A. \& S. Hunt (1997): The Problem of Quality of Life in Medicine, Journal of the American Medical Association 278, 47-50.

Lukes, S. (1986): Power, Oxford: Basil Blackwell.

Lykken, D. \& A. Tellegren (1996): Happiness is a Stochastic Phenomenon. Psychological Science 7 (3), 186-9.

McDowell, I. \& C. Newell (1987): Measuring Health, Oxford: Oxford University Press.

McGregor, J. A. (2000): A Poverty of Agency: Resource Management Amongst Poor People in Bangladesh (unpublished).

Michalos, A. C. (1985): Multiple Discrepancies Theory (MDT). Social Indicators Research 16, $347-413$

Michalos, A. C. (2001): Social Indicators Research and Health-Related Quality of Life Research. Plenary Session ISQOLS 2001 (unpublished).

Milde, F. K, L. K. Hart, P. S. Zehr (1992): Quality of Life of Pancreatic Transplant Recipients. Diabetes Care 15, 1459-63. 
O’Boyle, C., H. McGee, A. Hickey, K. O’Malley \& C. R. B. Joyce (1992): Individual Quality of Life in Patients Undergoing Hip Replacement. Lancet 339, 1088-91.

Ravallion, M. \& M. Lokshin (2001): Identifying Welfare Effects from Subjective Questions. Economica $68,335-57$.

Rosser, R., M. Cottee, R. Rabin\& C. Selai (1992): Index of Health-related Quality of Life. A. Hopkins (ed.): Measures of Quality of Life and the Uses to which such Measures May Be Put, London: RCP.

Ruta, D. A., A. M. Garratt, M. Leng, I. T. Russell, \& L. M. MacDonald (1994): A New Approach to the Measurement of Quality of Life. The Patient Generated Index. Medical Care 32 (11), 1109-26.

Ruta, D. (1997): Managing Health and Measuring Health Outcomes. Paper presented at: Managing and Measuring Health Outcomes: From Policy to Practice, Canberra: University of Wollongong.

Ryff, C. D. (1995): Psychological Well-being in Adult Life. Current Directions in Psychological Science 4 (4), 99-104.

Sartorius, N., W. Kuyken (1994): Translation of Health Instruments. J. Orley \& W. Kuyken (eds.): Quality of Life Assessment: International Perspectives, Heidelberg: Springer Verlag.

Schimmack, U., E. Diener \& S. Oishi (2002): Life-satisfaction is a Momentary Judgement and a Stable Personality Characteristic: The Use of Chronically Accessible and Stable Sources. Journal of Personality and Social Psychology 70 (3), 345-84.

Schwarz, N. \& F. Strack: Reports of Subjective Well-being: Judgemental Processes and their Methodological Implications. D. Kahneman, E. Diener \& N. Schwarz (eds.): Well-being: The Foundations of Hedonic Psychology, New York: Russell Sage Foundation, 61-84.

Skevington, S. \& K. O'Connell, K. (2003): Assessing the Importance of Quality of Life Using the WHOQuality of Life-100: How can we Identify Poor Quality of Life? (in press).

Strategy Unit (2002): Life Satisfaction: The State of Knowledge and Implications for Government, London: Cabinet Office.

Veenhoven, R. (1996): Happy Life-Expectancy. Social Indicators Research 39 (1), 1-58

Veenhoven, R. (2000): The Four Qualities of Life. Ordering Concepts and Measures of the Good Life. Journal of Happiness Studies 1, 1-39.

Ware, J. E., C. D. Sherbourne (1992): The MOS Short form 36-item health survey (SF-36): Conceptual Framework and Item Selection. Medical Care 30, 473-83.

WHO-Quality of Life Group (1995): The World Health Organisation Quality of Life assessment (WHOQuality of Life): Position Paper from the World Health Organisation. Social Science and Medicine 41, 1403-9.

WHO-Quality of Life Group (1998): The WHO Quality of Life Assessment (WHO-Quality of Life): Development and General Psychometric Properties. Social Science and Medicine 46, 1569-85.

Williams, J. I. (2000): Ready, Set, Stop and Standing. Journal of Clinical Epidemiology, 53, pp. 25-7. 